\title{
Atitudes de empresários do Sul do Brasil em relação aos portadores de doenças mentais
}

\author{
Businessmen attitudes regarding mental disodered \\ individuals in the South of Brazil \\ Dalnei Minuzzi Delevati', Lílian dos Santos Palazzo’
}

\section{Palavras-chave}

Atitudes, transtorno mental, trabalho, reinserção social.

\section{Keywords}

Attitudes, mental disorder, work, social reinsertion.

O trabalho é reconhecidamente importante veículo de inclusão social e exercício de cidadania. Entretanto, poucas oportunidades são oferecidas àqueles portadores de transtornos mentais. Objetivo: Este estudo objetivou conhecer as atitudes de empresários, em relação aos portadores de transtornos mentais, filiados ao Centro Empresarial, em uma cidade do Rio Grande do Sul, Brasil $(n=536)$. Método: Delineamento transversal censitário. Foram estudadas as variáveis sociodemográficas e as atitudes diante dos portadores de transtornos mentais. Como instrumento de medida, foi utilizado questionário, anônimo, auto-aplicado, e a escala de atitudes e opiniões sobre a doença mental (ODM). $\mathrm{Na}$ análise dos dados foi utilizada estatística descritiva e ANOVA. Resultados: Encontrouse que $89,7 \%$ dos empresários denotam atitudes que caracterizam idéias protecionistas; $75,5 \%$ acreditam na irrecuperabilidade e na periculosidade dessas pessoas e 73,2\% manifestaram-se favoráveis à restrição social dos que sofrem de transtorno mental grave. Conclusão: Para efetiva reabilitação psicossocial do portador de transtorno mental, há necessidade de campanhas educativas com o intuito de sensibilizar os empresários para a inclusão laboral dessa população.

\section{ABSTRACT}

It is recognized that work is an important vehicle of social inclusion and for the exercise of citizenship. However, there are few opportunities for people with mental disorders. Objective: This study aimed to find out businessmen's attitudes to mental disorders. These businessmen are members of a Business Center in a town in Rio Grande do Sul, Brazil ( $n=536)$. Method: A crosssectional census study. Sociodemographic variables and attitudes towards mental disorders were study. The measurement instrument employed was an anonymous, self-administered questionnaire and the Attitudes and Opinions Mental IIIness Scale (ODM). The data were analyzed using descriptive statistics and ANOVA. Results: It was found that $89.7 \%$ indicated attitudes that characterize protectionist ideas; $75.5 \%$ believe these people are irredeemable and dangerous and $73.2 \%$ indicated that they were in favor of the social restriction of people with mental disorders. Conclusions: In order to achieve effective psychosocial rehabilitation of the mentally disorders, it is necessary to carry out educational campaigns with the aim of persuading businesspeople to include this population in their workforce.

1 Saúde Coletiva da Universidade Luterana do Brasil (Ulbra).

Recebido em

16/5/2008

Aprovado em

28/10/2008
Endereço para correspondência: Dalnei Minuzzi Delevati Rua General Canabarro, 598/03 - 97700000 - Santiago, RS E-mail:dmdelevati@bol.com.br 
Segundo a Organização Mundial da Saúde (OMS), cerca de 450 milhões de pessoas, no mundo, padecem de enfermidades neuropsiquiátricas, atingindo prevalência pontual ao redor de 10\%. Além disso, aproximadamente, 24\% de todos os pacientes, atendidos por profissionais de atenção primária, têm um ou mais transtornos mentais'.

O movimento da reforma psiquiátrica no Brasil propiciou o avanço nas políticas públicas de saúde mental, destacando a importância da reabilitação psicossocial das pessoas portadoras de transtornos mentais, visando a melhora da sua qualidade de vida e a de seus familiares. A reabilitação caracteriza-se por ser processo de reconstrução; exercício pleno da cidadania e também de plena contratualidade nos três grandes cenários da vida: habitat, rede social e trabalho com valor social ${ }^{2}$.

As estratégias de reabilitação psicossocial variam conforme as necessidades do usuário, o contexto no qual é desenvolvida (hospital ou comunidade) e as condições culturais e socioeconômicas do país onde é efetivada. Os principais objetivos são: a emancipação do usuário, a redução da violência e da discriminação e a reinserção socia| ${ }^{3,4}$. Neste sentido, o trabalho fornece não somente a recompensa monetária, mas, também, benefícios não-financeiros, que incluem o sentimento de identidade e o desenvolvimento de oportunidades, para as pessoas com transtornos mentais, de participação na sociedade como cidadãos ativos 5 .

De acordo com o relatório da OMS, é possível encontrar, ao redor do mundo, milhares de bons exemplos de pessoas com transtornos mentais, não somente integradas às respectivas comunidades, mas desempenhando, de modo efetivo, papéis produtivos e economicamente importantes. Somente na Europa, cerca de 10 mil indivíduos em tal situação estão trabalhando em negócios e empresas que foram criadas para Ihes dar empregos'.

Em estudo de revisão sobre o assunto, foi observado que os portadores de transtornos mentais que trabalhavam apresentaram melhor qualidade de vida, quando comparados aos que não exerciam atividade laboral. Tal associação foi evidenciada, sobretudo, considerando os domínios de satisfação com a situação empregatícia e financeira ${ }^{6}$. Entretanto, o que se verifica, na realidade, é que muitas pessoas com transtornos mentais estão excluídas do mercado de trabalho, embora a maioria manifeste o desejo de realizar atividade labora| ${ }^{5,7}$. Entre as diversas barreiras encontradas estão as atitudes dos empregadores, freqüentemente associadas à discriminação, ao medo da loucura e da periculosidade ${ }^{8}$, à idéia de que são pessoas incapazes para o trabalho ${ }^{9}$ à crença de que apresentam baixa produtividade?.

Diante do exposto, concentrar especial atenção ao segundo setor é indiscutivelmente importante, já que os empresários representam a porta de entrada para o mercado de trabalho. Portanto, o presente estudo tem como objetivo conhecer as atitudes dos empresários em relação aos portadores de transtornos mentais e examinar a possível associação com fatores sociodemográficos.

\section{MÉTODO}

Trata-se de estudo epidemiológico transversal censitário, realizado com todos os empresários ativos, cadastrados no centro empresarial de uma cidade do Rio Grande do Sul. O município localiza-se a $440 \mathrm{~km}$ da capital do estado e apresenta população de 49.558 habitantes $^{10}$. Está geograficamente inserido em uma região carente de desenvolvimento industrial. Apresenta o êxodo rural como uma de suas principais preocupações na esfera social. Entre as atividades econômicas predomina o comércio varejista e o segundo ramo mais importante é o agronegócio, destacando-se na produção de gado de corte, seguida de culturas agrícolas, como soja, milho, feijão e sorgo. Também se destaca por possuir oito unidades militares, percentual significativo de servidores públicos na população.

A variável dependente está constituída pelas atitudes dos empresários diante dos portadores de transtornos mentais. As variáveis independentes foram sociodemográficas, como sexo, idade, estado civil, escolaridade, ramo de atividade e porte da empresa. Para caracterizar essa última, foram considerados os critérios da Secretaria da Fazenda do estado do Rio Grande do Sul". O estudo foi aprovado pelo Comitê de Ética da Universidade Luterana do Brasil (Ulbra), Canoas/RS (2006-382H).

Como instrumento de medida, utilizou-se um questionário, anônimo, auto-aplicado e organizado em duas partes: a primeira fornece informações sociodemográficas e a segunda, está constituída pela escala de atitudes e opiniões sobre a doença mental (ODM), traduzida e validada para o Brasil'12. Essa escala foi criada por Cohen e Struening em $1962^{13}$ Opinions about mental illness ideology scale (Opiniões sobre a doença mental). Optou-se pelo uso desta escala porque, após revisão na literatura sobre o assunto, não se encontrou outro instrumento, validado para o Brasil, que pudesse responder aos objetivos do estudo. A escala é formada por sete fatores, a seguir especificados:

- autoritarismo: contém idéias de irrecuperabilidade e periculosidade do portador de transtorno mental (questões: 21, 26, 31, 32, 34, 36, 40, 45, 49);

- benevolência: contém idéias protecionistas e paternalistas (questões: 12, 18, 22, 23, 38);

- ideologia da higiene mental: abrange idéias de que o portador de transtorno mental é igual às outras pessoas, diferindo, apenas, qualitativamente (questões: 2, 3, 8, 13, $27,28,33,41,44,47,50)$;

- restrição social: abrange idéias de que o portador de transtorno mental deve ter seus direitos pessoais e sociais restritos (questões: 4, 14, 19, 24, 37, 39, 51); 
- etiologia interpessoal: idéias de que o transtorno mental origina-se a partir de problemas interpessoais (questões: 5, 10, 20, 25, 30, 35);

- etiologia do esforço mental: idéias de que o transtorno mental originou-se de excessivo esforço cerebral (questões: 1, 7, 9, 15, 16, 43, 46, 48);

- visão minoritária: idéias de que o portador de transtorno mental é diferente em aparência externa (questões: 6, $11,17,29,42)$.

Assim, a escala ODM é composta de 51 questões, cada uma delas com seis alternativas do tipo LIKERT, seguindo a seqüência progressiva de seis pontos de concordância, desde "concordo totalmente" até "discordo totalmente". Essas afirmações estão agrupadas em sete fatores, segundo a fórmula para a correção da escala ${ }^{12,13}$. Após serem efetuadas as contagens dos pontos, chega-se ao escore bruto para cada fator. Como os fatores da ODM são compostos por números diferentes de itens, e sem conversões, pode-se ter um fator com valor elevado e outro com valor baixo, tornando difícil a apresentação. Assim, se aplicará à transformação em escores Sten. Tal padronização de valores é para simplificar a apresentação e a análise dos resultados encontrados. Portanto, a transformação em escores Sten propiciará comparação padrão entre os fatores com variação entre 1 a 10, exceto benevolência, cuja variação é de 1 a $8^{14}$.

A equipe de aplicadores foi devidamente treinada pelo coordenador do estudo, em relação aos objetivos do estudo, abordagem dos empresários. O questionário foi pré-testado em um estudo-piloto com um grupo de 50 empresários da cidade vizinha ao município em que foi desenvolvido o estudo. O estudo-piloto também serviu para complementar e avaliar o treinamento dos 16 estudantes universitários dos cursos de psicologia e enfermagem da Universidade Regional Integrada (URI) do Alto Uruguai e das Missões, campus Santiago.

A coleta de dados foi realizada de maneira sistemática, em que, de posse de documento autorizando a realização da pesquisa e de lista com o nome e endereço comercial fornecida pelo centro empresarial do município, cada empresário foi visitado e convidado a participar do estudo. Após a assinatura do termo de consentimento livre e esclarecido, o questionário prenchido pelo empresário foi depositado em uma urna, para garantir o anonimato.

O número total de empresários filiados ao centro empresarial na condição de ativos era 630, porém 20 empresas (3,2\%) não estavam mais em atividade, sendo excluídas do estudo. Dos 610 incluídos, houve perda de 47 sujeitos (7,7\%), assim discriminada: 33 (5,2\%) não aceitaram participar da pesquisa e 14 (2,2\%) não foram encontrados nos endereços declarados, resultando 563 indivíduos estudados.

O controle de qualidade foi realizado por meio de contato telefônico com 5\% dos empresários que responderam ao questionário. Após a conclusão da codificação dos questionários preenchidos, os dados foram duplamente digitados em arquivos separados no Epidata 3.1 e comparados em busca de possíveis erros de digitação. Na seqüência, foram devidamente corrigidos, e colocados no programa SPSS 10.0. para análise. Os dados foram analisados por meio de estatística descritiva para o estudo da distribuição das freqüências e ANOVA para comparação das médias complementadas pelo teste de Tukey, que visa a identificar quais as médias que, tomadas duas a duas, diferem significativamente entre si. O nível de significância utilizado foi de 5\%. Para se conhecer a distribuição dos sujeitos, dentro de cada fator, naqueles cuja variação foi de 1 a 10, escolheu-se o valor 5 como referência (metade). Para benevolência, que vai de 1 a 8, o valor foi 4. Isso proporcionou saber o percentual de sujeitos que estava mais próximo ou mais afastado do ponto máximo de cada fator.

\section{RESULTADOS}

Na Tabela 1, apresenta-se a distribuição das variáveis sociodemográficas. Assim, a maioria dos participantes era do sexo masculino (55,1\%), casados (72,9\%), na faixa etária entre 40 e 49 anos (31,3\%); 48,8\% cursaram o nível médio e 36,5\% o nível superior. Trezentos e oitenta e seis empresas (68,8\%) pertenciam ao ramo comercial, sendo 360 (64,3\%) classificadas microempresas.

Ao verificar o perfil de atitude dos empresários (Tabela 2 e Figura 1), observou-se que benevolência foi o fator de maior pontuação $(\bar{x}=5,97$; $D P=1,32)$, indicando atitudes protecionistas diante do transtorno mental e do seu portador. O fator autoritarismo ( $\bar{x}=6,84$; $\mathrm{DP}=1,85)$ foi prevalente, denotando atitudes que caracterizam a idéia de irrecuperabilidade e periculosidade. Com referência ao fator de etiologia do esforço mental $(\bar{x}=6,52 ; D P=1,82)$, os empresários entendem que o transtorno mental é resultado de um excessivo esforço cerebral. Os empresários manifestam-se favoráveis à restrição social ( $\bar{x}=6,69 ; \mathrm{DP}=1,68)$. Já em relação à visão minoritária $(\bar{x}=6,23 ; D P=1,71)$, apresentam a idéia de que o portador de transtorno mental é diferente em aparência externa. Relativamente à etiologia interpessoal $(\bar{x}=4,63 ; D P=1,84)$, os empresários acreditam que os transtornos mentais têm origem em problemas interpessoais. Por fim, referindo-se à ideologia da higiene mental $(\bar{x}=3,89 ; \mathrm{DP}=1,72)$, os entrevistados entendem que $\mathrm{O}$ portador de transtorno mental é igual às outras pessoas.

A Tabela 3 descreve a associação entre as variáveis sociodemográficas e a escala ODM. Os resultados revelaram que o sexo não se associou com nenhum fator. Quanto à faixa etária, a medida que aumenta a idade, aumentam os escores dos fatores benevolência $(p=0,046)$, restrição social 
Tabela 1. Caracterização das variáveis sociodemográficas da população estudada em empresários de uma cidade do Rio Grande do Sul, 2007 ( $n=563)$.

\begin{tabular}{lc}
\hline Caracteristicas* & $\mathbf{n}(\%)$ \\
\hline Sexo & \\
Masculino & $310(55,3)$ \\
Feminino & $251(44,7)$ \\
Faixa etária (anos) & \\
$18-30$ & $143(25,4)$ \\
$31-39$ & $139(24,7)$ \\
$40-49$ & $176(31,3)$ \\
$\geq 50$ & $105(18,7)$ \\
Escolaridade & \\
Nenhum/Fundamental & $83(14,8)$ \\
Médio & $274(48,8)$ \\
Superior & $205(36,5)$ \\
Estadocivil & \\
Solteiro & \\
Casado & $96(17,1)$ \\
Separado & $409(72,9)$ \\
Viúvo & $45(8,0)$ \\
Ramoda empresa & $11(2,0)$ \\
Comércio & \\
Indústria & $386(68,8)$ \\
Serviços & $11(2,0)$ \\
Porteda empresa & $164(29,2)$ \\
Micro & \\
Pequeno porte & \\
Geral & $360(64,3)$ \\
\hline & $145(25,9)$ \\
Notas & $55(9,8)$ \\
\hline
\end{tabular}

* Os totais diferem em virtude da não-ocorrência de respostas, em alquns casos.

Tabela 2. Pontuação dos fatores da escala ODM em empresários de uma cidade do Rio Grande do Sul, 2007 ( $n=563$ ).

\begin{tabular}{lccccc}
\hline Fatores*(Sten) & Média & Desvio-padrão & Mediana & Mínimo & Máximo \\
\hline Autoritarismo & 6,84 & 1,85 & 7,00 & 1,00 & 10,00 \\
Benevolência & 5,97 & 1,32 & 6,00 & 1,00 & 8,00 \\
Ideologia da higiene mental & 3,89 & 1,72 & 4,00 & 1,00 & 10,00 \\
Restrição social & 6,69 & 1,68 & 7,00 & 1,00 & 10,00 \\
Etiologia interpessoal & 4,63 & 1,84 & 5,00 & 1,00 & 10,00 \\
Etiologia do esforço mental & 6,52 & 1,82 & 7,00 & 1,00 & 10,00 \\
Visão minoritária & 6,23 & 1,71 & 6,00 & 1,00 & 10,00 \\
\hline
\end{tabular}

*A variaçăo de respostas dos fatores pelo método de Sten é de 1 a 10, exceto para benevolência, que é de 1 a 8.

$(p=0,024)$ e crença na etiologia interpessoal do transtorno mental $(p=0,004)$.

A escolaridade apresentou associação, em que quanto maior o nível de estudo alcançado, menores foram os escores nos fatores autoritarismo ( $p=0,001)$, restrição social $(p=0,001)$, etiologia interpessoal $(p=0,011)$, etiologia do esforço mental $(p=0,001)$ e visão minoritária $(p=0,001)$. Já o estado civil, associou-se apenas com o fator visão minoritária ( $p=0,041$ ), demonstrando que os sujeitos viúvos, seguidos dos casados apresentaram escores mais elevados em relação aos solteiros e aos separados, acreditam que os que sofrem de transtorno mental podem ser facilmente identificados por sua aparência, seu modo de comportar-se ou vestir-se.

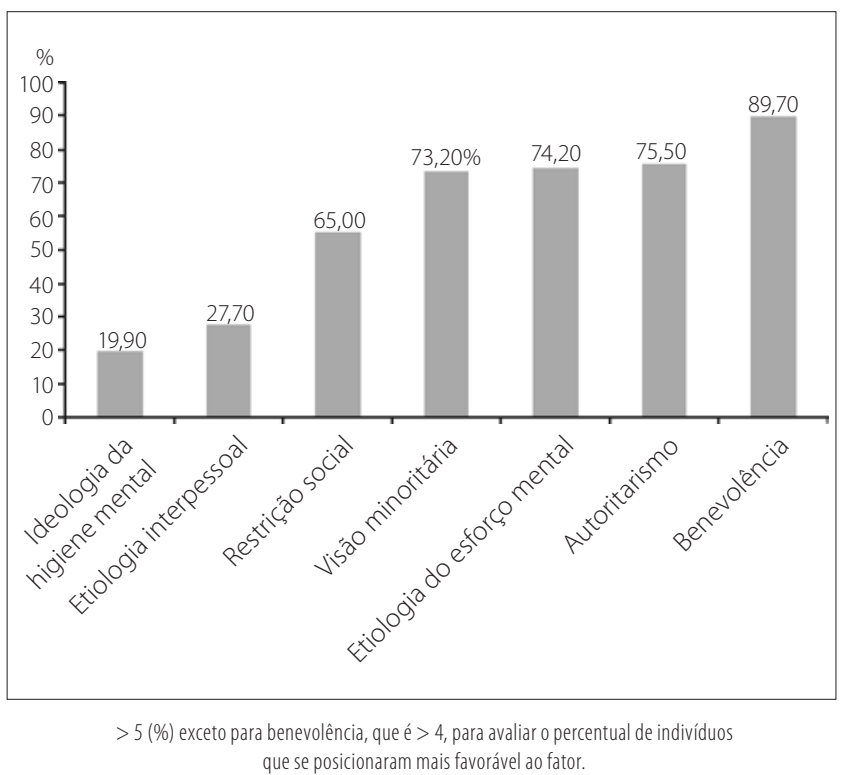

Figura 1. Distribuição dos sujeitos dentro de cada fator da escala ODM. Empresários de uma cidade do Rio Grande do Sul, 2007 ( $n=563)$.

Ao se estudar os empresários de acordo com as características da empresa e sua relação com os fatores da escala, foi encontrada associação com o ramo; o fator benevolência, apresenta escores mais baixos entre os industriais $(p=0,021)$. Já em relação ao porte da empresa, microempresários apresentaram escores significativamente mais elevados nos fatores benevolência $(p=0,038)$ e visão minoritária $(p=0,003)$ do que empresários de empresa geral e de pequeno porte.

\section{DISCUSSÃO}

A atenção ao portador de transtorno mental prevê que se tenha apoio nos Centros de Atenção Psicossocial (CAPS), onde deve receber atenção integral. Entretanto, apenas pequena parte da população recebe a assistência devida. Entre os vários empecilhos, destaca-se a violação aos direitos humanos, a carência de moradia e emprego, o acesso à saúde, a exclusão e o estigma. Estes interferem, significativamente, na detecção da doença, na adesão ao tratamento, na reabilitação e na reinserção socia|15,16.

Visando a contribuir para o entendimento das barreiras à viabilização do trabalho como instrumento de inclusão social, dos que padecem de transtorno mental, este estudo propôs avaliar o que pensam aqueles que gerenciam o mercado de trabalho. Entretanto, tem como principal limitação o fato de ter sido realizado com um grupo de empresários de uma cidade do interior do Rio Grande do Sul, podendo os dados ser estendidos apenas a empregadores de comunidades semelhantes. Ainda assim, permite embasar o diálogo com esse setor, para que possam ser criadas 
Tabela 3. Associação entre as variáveis sociodemográficas e os fatores da escala ODM em empresários de uma cidade do Rio Grande do Sul, $2007(n=563)$.

\begin{tabular}{|c|c|c|c|c|c|c|c|}
\hline Variáveis & $\begin{array}{c}\mathrm{F} 1 \\
\text { Média } \pm \mathrm{DP}\end{array}$ & $\begin{array}{c}\text { F2 } \\
\text { Média } \pm D P\end{array}$ & $\begin{array}{c}\text { F3 } \\
\text { Média } \pm \text { DP }\end{array}$ & $\begin{array}{c}\text { F4 } \\
\text { Média } \pm \text { DP }\end{array}$ & $\begin{array}{c}\text { F5 } \\
\text { Média } \pm \text { DP }\end{array}$ & $\begin{array}{c}\text { F6 } \\
\text { Média } \pm \text { DP }\end{array}$ & $\begin{array}{c}\text { F7 } \\
\text { Média } \pm \text { DP }\end{array}$ \\
\hline \multicolumn{8}{|l|}{ Sexo } \\
\hline Masculino & $6,98 \pm 1,72$ & $5,93 \pm 1,23$ & $3,80 \pm 1,66$ & $6,75 \pm 1,64$ & $4,65 \pm 1,85$ & $6,59 \pm 1,71$ & $6,25 \pm 1,56$ \\
\hline Feminino & $6,69 \pm 1,97$ & $6,03 \pm 1,40$ & $3,97 \pm 1,78$ & $6,61 \pm 1,72$ & $4,61 \pm 1,83$ & $6,44 \pm 1,93$ & $6,22 \pm 1,87$ \\
\hline$p$ & 0,069 & 0,348 & 0,247 & 0,309 & 0,309 & 0,310 & 0,788 \\
\hline \multicolumn{8}{|l|}{ Faixa etária } \\
\hline $18-30$ & $6,73 \pm 1,84$ & $5,79^{a} \pm 1,47$ & $3,83 \pm 1,82$ & $6,53^{a} \pm 1,57$ & $4,47^{a} \pm 1,72$ & $6,49 \pm 1,71$ & $6,08 \pm 1,67$ \\
\hline $31-39$ & $6,71 \pm 1,72$ & $5,86^{b} \pm 1,38$ & $3,91 \pm 1,72$ & $6,60^{b} \pm 1,74$ & $4,32^{\mathrm{a}} \pm 1,71$ & $6,45 \pm 1,82$ & $6,22 \pm 1,64$ \\
\hline $40-49$ & $6,84 \pm 1,90$ & $6,06^{b} \pm 1,27$ & $3,91 \pm 1,59$ & $6,61^{b} \pm 1,72$ & $4,71^{b} \pm 1,89$ & $6,38 \pm 1,92$ & $6,23 \pm 1,73$ \\
\hline$\geq 50$ & $7,14 \pm 1,93$ & $6,21^{c} \pm 1,08$ & $3,89 \pm 1,78$ & $7,1^{c} \pm 1,61$ & $5,12^{\complement} \pm 1,97$ & $6,89 \pm 1,76$ & $6,45 \pm 1,80$ \\
\hline$p$ & 0,267 & $0,046^{\mathrm{a}<\mathrm{b}<c}$ & 0,976 & $0,024^{a<b<c}$ & $0,004^{a<b<c}$ & 0,130 & 0,432 \\
\hline \multicolumn{8}{|l|}{ Escolaridade } \\
\hline Até fundamental & $7,00^{b} \pm 1,79$ & $6,22 \pm 1,18$ & $4,07 \pm 1,74$ & $7,05^{b} \pm 1,55$ & $4,96^{b} \pm 2,21$ & $7,07^{\mathrm{b}} \pm 1,78$ & $6,63^{b} \pm 1,55$ \\
\hline Médio & $7,11^{b} \pm 1,85$ & $5,98 \pm 1,21$ & $3,72 \pm 1,65$ & $6,87^{b} \pm 1,66$ & $4,75^{b} \pm 1,75$ & $6,72^{b} \pm 1,73$ & $6,41^{b} \pm 1,80$ \\
\hline Superior & $6,42^{\mathrm{a}} \pm 1,79$ & $5,86 \pm 1,47$ & $4,02 \pm 1,78$ & $6,31^{a} \pm 1,68$ & $4,34^{a} \pm 1,75$ & $6,03^{\mathrm{a}} \pm 1,84$ & $5,84^{a} \pm 1,57$ \\
\hline $\mathrm{p}$ & $<0,001^{\mathrm{b}>\mathrm{a}}$ & 0,115 & 0,096 & $<0,001^{\mathrm{b}>\mathrm{a}}$ & $0,011^{b>a}$ & $<0,001^{\mathrm{b}>\mathrm{a}}$ & $<0,001^{b>a}$ \\
\hline \multicolumn{8}{|l|}{ Estado civil } \\
\hline Solteiro & $6,64 \pm 1,65$ & $5,89 \pm 1,47$ & $3,95 \pm 1,72$ & $6,43 \pm 1,57$ & $4,29 \pm 1,57$ & $6,38 \pm 1,69$ & $5,91^{\mathrm{a}} \pm 1,49$ \\
\hline Casado & $6,91 \pm 1,87$ & $6,03 \pm 1,25$ & $3,90 \pm 1,69$ & $6,72 \pm 1,68$ & $4,76 \pm 1,87$ & $6,55 \pm 1,81$ & $6,33^{b} \pm 1,69$ \\
\hline Separado & $6,71 \pm 2,03$ & $5,56 \pm 1,45$ & $3,60 \pm 1,71$ & $6,84 \pm 1,91$ & $4,40 \pm 2,10$ & $6,58 \pm 2,02$ & $5,96^{c} \pm 2,12$ \\
\hline Viúvo & $6,73 \pm 2,00$ & $6,27 \pm 1,35$ & $3,45 \pm 2,38$ & $7,27 \pm 1,56$ & $4,09 \pm 1,38$ & $6,72 \pm 2,15$ & $7,00^{c} \pm 1,84$ \\
\hline $\mathrm{p}$ & 0,554 & 0,092 & 0,554 & 0,243 & 0,078 & 0,824 & $0,041^{a<b<c}$ \\
\hline \multicolumn{8}{|l|}{ Ramo da empresa } \\
\hline Comércio & $6,85 \pm 1,89$ & $5,98^{\mathrm{b}} \pm 1,35$ & $3,86 \pm 1,71$ & $6,68 \pm 1,66$ & $4,63 \pm 1,86$ & $6,61 \pm 1,84$ & $6,31 \pm 1,72$ \\
\hline Indústria & $6,82 \pm 1,66$ & $4,91^{\mathrm{a}} \pm 1,22$ & $3,45 \pm 0,93$ & $7,18 \pm 1,54$ & $4,82 \pm 2,32$ & $6,36 \pm 2,34$ & $5,45 \pm 1,57$ \\
\hline Serviços & $6,82 \pm 1,77$ & $6,04^{b} \pm 1,21$ & $3,95 \pm 1,77$ & $6,66 \pm 1,73$ & $4,61 \pm 1,78$ & $6,31 \pm 1,73$ & $6,13 \pm 1,66$ \\
\hline $\mathrm{p}$ & 0,982 & $0,021^{\mathrm{a}<\mathrm{b}}$ & 0,622 & 0,612 & 0,935 & 0,200 & 0,160 \\
\hline \multicolumn{8}{|l|}{ Porte da empresa } \\
\hline Micro & $6,93 \pm 1,80$ & $6,08^{\mathrm{a}} \pm 1,30$ & $3,78 \pm 1,78$ & $6,81 \pm 1,69$ & $4,68 \pm 1,87$ & $6,61 \pm 1,87$ & $6,41^{\mathrm{a}} \pm 1,70$ \\
\hline Pequeno & $6,76 \pm 1,95$ & $5,78^{b} \pm 1,34$ & $4,02 \pm 1,56$ & $6,47 \pm 1,60$ & $4,67 \pm 1,84$ & $6,31 \pm 1,73$ & $6,01^{b} \pm 1,71$ \\
\hline Geral & $6,60 \pm 1,83$ & $5,80^{\mathrm{b}} \pm 1,27$ & $4,15 \pm 1,73$ & $6,47 \pm 1,71$ & $4,27 \pm 1,59$ & $6,53 \pm 1,70$ & $5,69^{b} \pm 1,65$ \\
\hline$p$ & 0,376 & $0,038^{a>b}$ & 0,181 & 0,073 & 0,305 & 0,257 & $0,003^{a>b}$ \\
\hline
\end{tabular}

a,b, Letras iguais não diferem pelo teste de Tukey; F1: autoritarismo; F2: benevolência; F3: ideologia da higiene mental; F4: restrição social; F5: etiologia interpessoal; F6: etiologia do esforço mental; F7: visão minoritária.

alternativas reais e concretas de participação laboral dos portadores de transtorno mental, em vez de aceitar que a sociedade continue excluindo o diferente.

Observou-se que a atitude do empresariado em relação ao transtorno mental apresenta-se, principalmente, com características de protecionismo e paternalismo, em que o portador de transtorno é visto como infeliz e, portanto, deve ser amparado. Soma-se a isso a noção de irrecuperabilidade pessoal e social, a periculosidade, que o transtorno mental é diferente das demais transtornos e o conceito de que o portador de transtorno é muito diferente das pessoas normais. Estes achados corroboram o encontrado em estudo realizado por Gaebel et al. ${ }^{17}$ em seis cidades de grande porte da Alemanha, sobre as atitudes em relação ao transtorno mental. Neste, a população entrevistada acreditava que os portadores de esquizofrenia eram violentos e perigosos ao público; cerca de 10\% admitiu ficar receosa de falar com alguém com esquizofrenia; 16\% afirmaram que sentiriam o próprio rendimento perturbado ao lado de alguém com transtorno mental; mais de $40 \%$ se sentiriam contrários a compartilhar o quarto com uma pessoa com esquizofrenia (tal como dentro do hospital) e 70\% não casariam com alguém portador de transtorno mental. Segundo Foucault ${ }^{18}$, este tipo de concepção era a vigente na Idade Média, em que o louco não se enquadrava às regras sociais e por isso era considerado improdutivo. Aduzia que a segregação está associada ao fato de que o louco representa perigo iminente à sociedade e esse rótulo de perigoso leva a respostas, como o medo, a indiferença e a exclusão $0^{19,20}$. Assim, é possível que o perfil de atitudes observado no grupo estudado reflita a lenta evolução da representação social do transtorno mental até a atualidade.

Verificou-se associação entre o aumento da idade e maiores escores de atitudes paternalistas e assistencialistas, com idéias de que o portador de transtorno mental deve ficar isolado dos demais, perdendo, inclusive, seus direitos como cidadão, e com pensamentos de que o transtorno mental se origina de dificuldades nos relacionamentos interpessoais. Talvez o temor, não se fundamente somente na realidade, mas no medo da loucura, particularidade que todo ser humano traz consigo e que, possivelmente, venha a refletir-se na atitude diante do portador de transtorno mental, fazendo que predominem sentimentos negativos ${ }^{8}$. Nesse sentido, estudo realizado na Alemanha mostra que aqueles com idade avançada tiveram menos conhecimento sobre esse tipo de transtorno e maior distância social ${ }^{17}$. Por 
essa razão, alguns autores preconizam ações de educação em saúde mental dirigidas a crianças e adolescentes, no sentido de aprimorar-lhes a compreensão sobre o transtorno mental, para que quando cheguem na idade adulta, atuem de maneira menos discriminatória ${ }^{21}$.

O temor ao diferente é possível que também se veja refletida nos sujeitos viúvos e casados, cuja condição remete a maior responsabilidade e preocupação, sobretudo com filhos, mantendo atitudes de segregação pela aparência externa. Cabe ressaltar que o número de viúvos estudado era muito pequeno (apenas 2\%), e que talvez número maior poderia modificar o resultado.

Os sujeitos menos escolarizados apresentaram atitudes relacionadas à postura autoritária, visualizando o portador de transtorno mental como uma pessoa sem chances de recuperação, agressiva e perigosa, reforçando o distanciamento social, o estigma e a discriminação ${ }^{17}$. Pode-se interpretar que a informação representa a grande aliada no processo de redução do preconceito. Estudo que utilizou a escala ODM mostrou que a instrução acadêmica pode influenciar favoravelmente a mudança das atitudes diante do transtorno mental e à pessoa que dele padece ${ }^{22}$.

No que tange ao ramo da empresa, os empresários do comércio e serviços apresentaram atitudes de protecionismo e paternalismo, ou seja, pensam que o portador de transtorno mental, por causa de sua infelicidade, deve ser amparado, com cuidados, atenção pessoal e conforto material. Em relação ao porte da empresa, os empresários do comércio e os microempresários apresentam atitude de protecionismo e paternalismo, bem como de restrição social. Pode-se compreender, assim, que tais atitudes configuram mecanismos de discriminação direta em relação ao portador de transtorno mental. Estudos mostram que muitos dos empregadores que não tinham admitido indivíduo portador de transtorno mental apresentaram, como justificativa, que estes ofereceriam riscos em relação à segurança, tanto para si mesmos quanto para os outros empregados ${ }^{7,8,23}$. Observa-se, assim, que há a crença de que os portadores de transtornos mentais são incapazes, improdutivos e com limitada capacidade de compreensão. Por outro lado, os pacientes esquizofrênicos que trabalham, incluindo trabalho assistido, apresentaram melhor qualidade de vida, quando comparados aos que não trabalhavam. A renda, recebida pelos pacientes esquizofrênicos, mostrou-se também associada (positivamente) à melhor qualidade de vida6,24. 0 medo de vivenciar resposta negativa, diante de solicitação de emprego, pode apresentar sérias conseqüências para o portador de transtorno mental, como significativa perda da auto-estima ${ }^{3,25}$. Segundo pesquisa, realizada no Chile, 68\% dos esquizofrênicos participantes estariam em condições de trabalhar, se lhes fossem dadas oportunidades ${ }^{9}$.

Considerando os resultados deste estudo, observa-se que os empresários apresentam atitudes, diante dos transtornos mentais e do seu portador, relacionadas à discriminação, ao estigma e ao preconceito. Cabe salientar que tais atitudes podem ser significativas e possivelmente colaboradoras da dificuldade para a reinserção laboral do portador de transtorno mental. Por isso, devem ser pensadas estratégias de intervenção com este grupo populacional que propiciem maior conhecimento sobre saúde mental, modificando a representação que tem sobre o transtorno mental.

\section{CONCLUSÃO}

Conclui-se que os empresários apresentam atitudes, em relação aos portadores de transtornos mentais, caracterizadas, principalmente, por idéias protecionistas, irrecuperabilidade e periculosidade, e favoráveis à restrição social. Possivelmente, são resultantes da desinformação, desconhecimento e preconceitos, os quais sustentam barreiras à plena integração dos portadores de transtornos mentais na sua comunidade e no mercado de trabalho. Assim, se faz necessário processo de intervenção nessa população empregadora. Tal intervenção poderá ser feita por meio de campanhas educativas, com o intuito de desmistificação e sensibilização dos empresários, no sentido de mudar a representação social que têm sobre os transtornos mentais, facilitando a reinserção laboral daqueles que apresentam transtorno mental. Além disso, as referidas campanhas devem contemplar, também, a promoção da saúde mental e a prevenção dos transtornos mentais.

\section{REFERÊNCIAS}

1. Ministério da Saúde. Secretaria de Atenção à Saúde. Departamento de Ações Programáticas Estratégicas. Coordenação Geral de Saúde Mental. Comunicação e Educação em Saúde. Brasília: MS; 2004.

2. Pitta A. 0 que é reabilitação psicossocial no Brasil, hoje? In: Pitta A, organizador. Reabilitação psicossocial no Brasil. São Paulo: Hucitec; 1996

3. Link BG, Struening EL, Neese S, Asmussen S, Phelan JC. Stigma as a barrier to recovery: the consequences of stigma for the self-esteem of people with mental illnesses. Psychiatr Serv. 2001;52(12):1621- 6 .

4. Berenstein I. Notas sobre la violencia. Psicoanálisis AP de BA. 2000; XXII(2):257-71.

5. Boardman J, Grove B, Perkins R, Shepherd G. Work and employment for people with psychiatric disabilities. Br J Psychiatry. 2003;182(6):467-8.

6. Souza LA, Coutinho ESF. Fatores associados à qualidade de vida de pacientes com esquizofrenia. Rev Bras Psiquiatr. 2006;28(1):50-8.

7. Scheid TL. The Americans with Disabilities Act, mental disability, and employment practices. J Behav Health Serv Res. 1998;25:312-4.

8. Thompson HA, Stuart H, Bland R, et al. Attitudes about schizofrenia from the pilot site of the WPA worldwide campaing against the stigma of schizophrenia. Soc Psychiatry Psychiatr Epidemiol. 2002;37(10):475-82.

9. Chuaqui J. El estigma en la esquizofrenia. Ciencias sociales online. Santiago, Chile: Universidad de Viña del Mar; 2005. p. 1.

10. Brasil. Instituto Brasileiro de Geografia e Estatística. Censo Demográfico. Brasília: IBGE; 2007. 
11. Serviço Brasileiro de Apoio às Micro e Pequenas Empresas (Sebrae/RS): Entenda o Simples gaúcho, Porto Alegre; 2006.

12. Rodrigues CR. Atitudes frente à doença mental: estudo transversal de uma amostra de profissionais da saúde. Faculdade de Medicina de Ribeirão Preto USP; 1983.

13. Cohen J, Struening E. Opinions about mental illness in the personnel of two large mental hospitals. J Abnorm Soc Psychol. 1962;64:349-60.

14. Canfield A. The sten scala: a modified C - scale. Educ Psychol Meas. 1951;11:295-7.

15. Ministério da Saúde. Saúde mental e economia solidária: inclusão social pelo trabalho. Brasília: MS; 2005

16. Ministério da Saúde. Saúde mental e economia solidária: inclusão social pelo trabalho. Relatório final do grupo de trabalho. Brasilia: MS; 2006.

17. Gaebel W, Baumann A, Zäske H. Intervening in a multi-level network: progress of the German open the doors projects. World Psychiatry. 2005;4(1):16-20.

18. Foucault M. Doença mental e psicologia. $6^{a}$ ed. Rio de Janeiro: Tempo Brasileiro; 2000.
19. Foucault M. História da loucura. São Paulo: Perspectiva; 2002.

20. Mann C, Himelein M. Factors associated with stigmatization of persons with mental illness. Psychiatr Serv. 2004;55(2):185-7.

21. Pinfold $V$, Stuart $H$, Thornicroft $G$, et al. Working with young people: the impact of mental health awareness programmes in schools in the UK and Canada. World Psychiatry. 2005;4(1):50-4.

22. Pedrão LJ, Avanci RC, Malaguti SE. Perfil das atitudes de alunos do curso de enfermagem frente à doença mental, antes da influência da instrução acadêmica, proveniente de disciplinas de área específica. Rev Latino-Am Enfermagem. 2002; 10:794-9.

23. Gold J, Goldberg R, Mcnary SW, Dixon LB, Lehman AF. Cognitive correlates of job tenure among patients with severe mental illness. Am J Psychiatry. 2002;159(8):1395-402.

24. Cook JA, Lehman AF, Drake R, McFarlane WR, Gold PB, Leff HS, et al. Integration of psychiatric and vocational services: a multisite randomized, controlled trial of supported employment. Am J Psychiatry. 2005;162(10):1948-56.

25. Saraceno B. Levan I. Kohn R. The public mental health significance of research on socio-economic factors in schizophrenia and major depression. World Psychiatry. 2005;4(3):181-5. 